\title{
Biomarkers improve mortality prediction by prognostic scales in community-acquired pneumonia
}

\author{
R Menéndez, ${ }^{1}$ R Martínez, ${ }^{1}$ S Reyes, ${ }^{1} \mathrm{~J}$ Mensa, ${ }^{2}$ X Filella, ${ }^{3}$ M A Marcos, ${ }^{4}$ A Martínez, \\ C Esquinas, ${ }^{5}$ P Ramirez, ${ }^{6}$ A Torres ${ }^{5}$
}

\section{See Editorial, p. 556}

${ }^{1}$ Servicio de Neumología. Universitary Hospital La Fe, Ciber de enfermedades respiratorias (CIBERES), Valencia, Spain;

${ }^{2}$ Servicio de Infecciosas, Hospital Clinic,

IDIBAPSBarcelona, Spain;

${ }^{3}$ Servicio de Bioquímica, Hospital Clinic, IDIBAPS

Barcelona, Spain; ${ }^{4}$ Servicio de Microbiología, Hospital Clinic,

IDIBAPS, Barcelona, Spain

${ }^{5}$ Servicio de Neumología.

Hospital Clinic, IDIBAPS, Ciber de enfermedades respiratorias (CIBERES), Barcelona, Spain;

${ }^{6}$ Unidad Cuidados Intensivos. Hospital La Fe, Valencia, Spain

Correspondence to:

Dr R Menéndez, Servicio de Neumología, Hospital

Universitario La Fe, Avda de

Campanar 21, 46009 Valencia,

Spain; rmenend@separ.es

Received 25 July 2008 Accepted 10 December 2008

Published Online First

6 January 2009

\begin{abstract}
Background: Prognostic scales provide a useful tool to predict mortality in community-acquired pneumonia (CAP). However, the inflammatory response of the host, crucial in resolution and outcome, is not included in the prognostic scales.
\end{abstract}

Methods: The aim of this study was to investigate whether information about the initial inflammatory cytokine profile and markers increases the accuracy of prognostic scales to predict 30-day mortality. To this aim, a prospective cohort study in two tertiary care hospitals was designed. Procalcitonin (PCT), C-reactive protein (CRP) and the systemic cytokines tumour necrosis factor $\alpha$ (TNF $\alpha)$ and interleukins IL6, IL8 and IL10 were measured at admission. Initial severity was assessed by PSI (Pneumonia Severity Index), CURB65 (Confusion, Urea nitrogen, Respiratory rate, Blood pressure, $\geqslant 65$ years of age) and CRB65 (Confusion, Respiratory rate, Blood pressure, $\geqslant 65$ years of age) scales. A total of 453 hospitalised CAP patients were included.

Results: The 36 patients who died (7.8\%) had significantly increased levels of IL6, IL8, PCT and CRP. In regression logistic analyses, high levels of CRP and IL6 showed an independent predictive value for predicting 30day mortality, after adjustment for prognostic scales. Adding CRP to PSI significantly increased the area under the receiver operating characteristic curve (AUC) from 0.80 to 0.85 , that of CURB65 from 0.82 to 0.85 and that of CRB65 from 0.79 to 0.85 . Adding IL6 or PCT values to CRP did not significantly increase the AUC of any scale. When using two scales (PSI and CURB65/CRB65) and CRP simultaneously the AUC was 0.88 .

Conclusions: Adding CRP levels to PSI, CURB65 and CRB65 scales improves the 30-day mortality prediction. The highest predictive value is reached with a combination of two scales and CRP. Further validation of that improvement is needed.

Community-acquired pneumonia (CAP) continues to be a worldwide health problem. Its annual incidence is $0.3-0.5 \%$ in the adult population, and mortality reaches $5-15 \%$ in admitted patients, thus representing the leading cause of death from infectious disease, ${ }^{1-3}$ as reported by the ERS (European Respiratory Society) ${ }^{2}$ and found in data from the USA. ${ }^{45}$ This justifies the interest in identifying prognostic factors and developing tools able to predict mortality. The publication during the 1990s of the Fine risk scale, the Pneumonia Severity Index (PSI) ${ }^{6}$ to predict mortality in CAP represented a significant advance, as also did the more recent CURB65 (Confusion, Urea nitrogen, Respiratory rate, Blood pressure, $\geqslant 65$ years of age) scale and its simplified version CRB65(Confusion,
Respiratory rate, Blood pressure, $\geqslant 65$ years of age). Both scales have been validated, ${ }^{8}{ }^{7}$ but they have some limitations and they are believed to evaluate best opposite severity spectrums. Both scales calculate at diagnosis the probability of death, but they do not assess the inflammatory response of the host, which is currently considered a key aspect in the prognosis of the patient. ${ }^{10}$

A new approach is to evaluate biological parameters, as an expression of the host's inflammatory response against the microorganism, in order to identify the defence capacity of the patient and to individualise the prognosis. In that response, the host produces an amount of proinflammatory and anti-inflammatory cytokines that depends on genetic polymorphisms, the microorganism and the initial severity of the disease..$^{10}$ Although these mediators have a beneficial effect, an excessive release of cytokines has been associated with deleterious effects such as hypotension, myocardial dysfunction, hypoperfusion of vital organs, lactic acidosis and higher mortality. ${ }^{10-12}$ Biological markers of infection and inflammation have raised much interest because of their ability to reflect the inflammatory response of the host more rapidly and more feasibly. Previous studies with C-reactive protein (CRP), procalcitonin (PCT) and, more recently, proadrenomedullin ${ }^{13}$ have shown their correlation with initial severity and with poor outcome.

Our hypothesis is that the inclusion of biological markers, as an expression of the systemic inflammatory response, would yield additional information and would be able to improve the 30-day mortality prediction of the two most used prognostic scales, the PSI and the CURB65/CRB65 scales, in patients hospitalised due to CAP. Therefore, we analysed the diagnostic value of the scales to predict mortality adding information on the cytokines interleukin 6 (IL6), IL8, IL10. tumour necrosis factor $\alpha(\mathrm{TNF} \alpha)$, and the markers CRP and PCT.

\section{PATIENTS AND METHODS}

A prospective longitudinal study was performed in patients with CAP consecutively hospitalised in two hospitals. The inclusion criteria were a new radiographic infiltrate and at least two compatible clinical symptoms. Exclusion criteria were admission within the previous 15 days, immunosuppressive treatment and/or steroids ( $>15 \mathrm{mg} /$ day), leucopenia $<1000 / \mathrm{mm}^{3}$ or neutropenia $<500 / \mathrm{mm}^{3}$ (except if attributable to CAP) and patients with do-not-resuscitate (DNR) orders. The study was approved by the Ethics Committees of the two 
hospitals involved and the patients signed an informed consent.

\section{Data collection}

Data were collected about age, gender, smoking and alcohol habits (>80 g/day), comorbid diseases such as chronic obstructive pulmonary disease (COPD), and cardiac, liver, renal or central nervous system disorders. Recorded clinical signs and symptoms were: cough, expectoration, pleuritic chest pain, dyspnoea, acute confusion, temperature, respiratory and heart rates, systolic and diastolic blood pressure and the presence of rales. The following analytical data were recorded: leucocyte count, sodium, potassium, serum creatinine, alanine aminotransferase (ALT)/aspartate aminotransferase (AST) and arterial blood gas analysis. The PSI, ${ }^{6}$ CURB65 and CRB65 were also recorded. ${ }^{14}$

Surviving patients underwent follow-up radiological and serological study after 30 days. The endpoint variable was death within 30 days of admission.

\section{Determinations of cytokines, PCT and CRP}

Blood samples were drawn on the first day. Blood was centrifuged, coded and frozen at $-80^{\circ} \mathrm{C}$ until subsequent analyses. The determination of IL6, IL8, IL10 and TNF $\alpha$ was performed with a commercial enzymoimmunoassay technique (BioSource, Nivelles, Belgium). The limits of detection were $3 \mathrm{pg} / \mathrm{ml}$ for $\mathrm{TNF} \alpha, 2 \mathrm{pg} / \mathrm{ml}$ for IL6, $0.7 \mathrm{pg} / \mathrm{ml}$ for IL8 and $1 \mathrm{pg} / \mathrm{ml}$ for IL10.

An immunoluminometric technique was used to measure PCT (Liaison Bhams PCT, DiaSorin, Saluggia, Italia) with a detection limit of $0.3 \mathrm{ng} / \mathrm{ml}$. CRP was measured using an immunoturbidimetric method employing a commercially available test (Bayer Diagnostics, Leverkusen, Germany) with an Advia 2400.

\section{Statistical analysis}

Statistical analysis was performed with the SPSS 15.0 software. The $\chi^{2}$ test was used for categorical variables, and the Student $t$ or Mann-Whitney U tests for continuous variables. Correlation was analysed using the Spearman's rho correlation analysis.

Multivariable logistic regression was performed to predict 30day mortality (dependent variable); independent variables were initial severity and cytokines, and the PSI scale was stratified as follows: classes I-II, III, IV and V. CURB65 was stratified as $0-1,2,3,4-5$ and CRB65 was stratified as $0-1,2,3-5$.

In order to calculate the diagnostic value of markers, cytokines, PSI and/or CURB65/CRB65 scales to predict 30day mortality, multivariable logistic regression analyses were performed with several combinations. First, the logistic regression models included CRP and PCT as independent variables, and were adjusted either for PSI, for the CURB65 or for the CRB65 scale. Next, cytokines were added to each model as independent variables and multivariable analyses were repeated. For each regression logistic model, the area under the receiver operating characteristic (ROC) curve (AUC) was calculated for 30-day mortality prediction. Statistical comparison of AUCs was conducted according to the Hanley and McNeil method ${ }^{15}$; $p$ values $<0.05$ were considered significant. For resulting models, sensitivity, specificity and positive and negative predictive values were calculated. The Hosmer and Lemeshow goodness-of-fit test was performed for each model. ${ }^{16}$

\section{RESULTS}

\section{Patient population}

During the study period, 480 patients were eligible; the study group comprised 453 patients (419 of them with complete results for all cytokines and markers) with a mean age of 67.3 (17.1) (mean (SD)) years. Mortality within 30 days was $7.9 \%$ (36 patients); 31 of them died during hospitalisation (in-hospital mortality $6.8 \%$ ). The main demographic characteristics, comorbidity and initial severity measured by the two prognostic scales (PSI and CURB65) are shown in table 1.

Patients who died were older, had more neurological diseases and higher initial severity, and more patients belonged to PSI class $\mathrm{V}$ and CURB65 class $>3$. The microorganisms most frequently found were: 81 Streptococcus pneumoniae (18\%), 17 Legionella pneumophila (4\%), 12 Staphylococcus aureus (3\%), 12 Haemophilus influenzae, 11 Pseudomonas aeruginosa, 6 Escherichia coli (1\%), 5 Mycoplasma pneumoniae and 28 other microorganisms. We found mixed aetiology in 26 patients: 15 of them had $S$ pneumoniae together with other microorganisms.

Table 1 Characteristics and initial severity of the groups with and without mortality

\begin{tabular}{|c|c|c|c|}
\hline \multirow[b]{3}{*}{ Characteristic } & \multicolumn{2}{|l|}{ 30-Day mortality } & \multirow[b]{3}{*}{ p Value } \\
\hline & Yes & No & \\
\hline & $n=36(7.9 \%)$ & $\mathrm{n}=417(92.1 \%)$ & \\
\hline Age, mean (SD) & $79(8)$ & $66(17)$ & 0.0001 \\
\hline Gender, F/M (\%) & $15 / 21(41.7 / 58.3)$ & $156 / 261(37.4 / 62.6)$ & 0.6 \\
\hline Long-term care facility, no (\%) & $7(19.4)$ & $17(4.1)$ & 0.0001 \\
\hline Smoking, no (\%) & $4(11.1)$ & $95(22.8)$ & 0.1 \\
\hline Alcohol, no (\%) & $3(8.3)$ & 48 (11.5) & 0.5 \\
\hline \multicolumn{4}{|l|}{ Comorbidity, no (\%) } \\
\hline Heart failure & $9(25)$ & $67(16.1)$ & 0.1 \\
\hline Renal failure & $2(5.6)$ & $23(5.5)$ & 0.9 \\
\hline Diabetes & $5(13.9)$ & $86(20.6)$ & 0.3 \\
\hline Liver disease & $1(2.8)$ & $11(2.6)$ & 0.9 \\
\hline COPD & $6(16.7)$ & $73(17.5)$ & 0.8 \\
\hline Neurological disease & $21(58.3)$ & $77(18.5)$ & 0.0001 \\
\hline Neoplasm & $4(11.1)$ & $15(3.6)$ & 0.03 \\
\hline PSI, no (\%) & & & 0.01 \\
\hline I & 0 & $48(11.5)$ & \\
\hline II & 0 & $73(17.5)$ & \\
\hline III & $2(5.6)$ & $93(22.3)$ & \\
\hline IV & $14(38.9)$ & $153(36.7)$ & \\
\hline V & $20(55.6)$ & $50(12)$ & \\
\hline CRB65, no (\%) & & & 0.01 \\
\hline 0 & $1(2.8)$ & $78(19)$ & \\
\hline 1 & $4(11.1)$ & $189(46)$ & \\
\hline 2 & $14(38.9)$ & $112(27.3)$ & \\
\hline 3 & $13(36.1)$ & $29(7.1)$ & \\
\hline 4 & $4(11.1)$ & $3(0.7)$ & \\
\hline \multicolumn{4}{|l|}{ CURB65, no (\%) } \\
\hline 0 & $1(2.8)$ & $63(15.1)$ & 0.01 \\
\hline 1 & $1(2.8)$ & $140(33.6)$ & \\
\hline 2 & $7(19.4)$ & $123(29.5)$ & \\
\hline 3 & $13(36.1)$ & $66(15.8)$ & \\
\hline 4 & $10(27.8)$ & $23(5.5)$ & \\
\hline 5 & $4(11.1)$ & $2(0.5)$ & \\
\hline ICU admission & $9(25.0)$ & $42(10.1)$ & 0.007 \\
\hline
\end{tabular}

COPD, chronic obstructive pulmonary disease; CRB65, Confusion, Respiratory rate, Blood pressure, $\geqslant 65$ years of age; CURB65, Confusion, Urea nitrogen, Respiratory rate, Blood pressure, $\geqslant 65$ years of age; $F$, female; ICU, Intensive Care Unit; $M$, male; PSI, Pneumonia Severity Index. 
Table 2 Median (25th to 75th percentile) of cytokines and markers according to initial severity

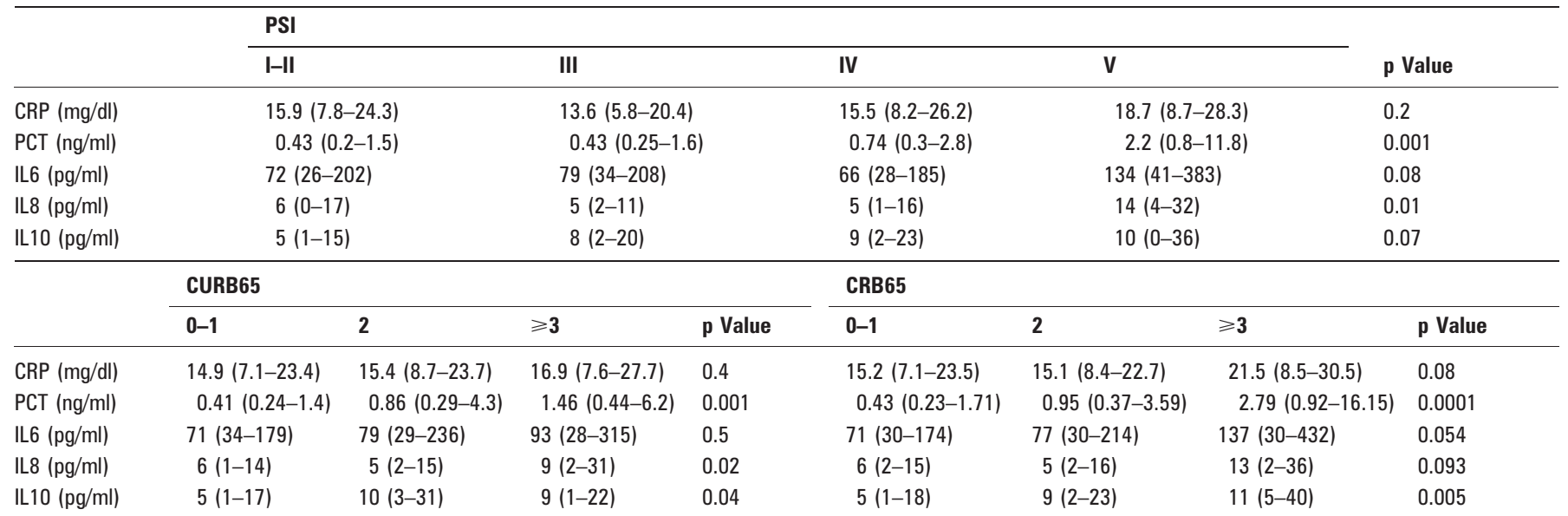

CRB65, Confusion, Respiratory rate, Blood pressure, $\geqslant 65$ years of age; CRP, C-reactive protein; CURB65, Confusion, Urea nitrogen, Respiratory rate, Blood pressure, $\geqslant 65$ years of age; IL, interleukin; PCT, procalcitonin; PSI, Pneumonia Severity Index.

\section{Initial severity, systemic cytokines and markers}

Results of cytokines and markers according to the prognostic scales are shown in table 2.

The medians were significantly higher in more severe disease for PCT and IL8 in both scales. We did not find any significant difference concerning prior treatment before admission or for any marker or cytokine.

A correlation analysis of cytokines and markers with initial severity showed that PCT had the highest positive correlation with PSI (rho 0.27, p<0.0001), CURB65 (rho 0.29, p<0.0001) and CRB65 (rho:0.26, p<0.0001) scales. IL8 also showed a low positive but significant correlation with CURB65 (rho 0.11, $\mathrm{p}<0.02$ ), and IL10 with PSI (rho $0.12 \mathrm{p}<0.01$ ), CURB65 (rho $0.11 \mathrm{p}<0.02$ ) and CRB65 (rho 0.1, p<0.001) scales. The correlation of the cytokines with CRP and PCT was studied. A positive correlation was found for CRP with IL6 (rho 0.55, $\mathrm{p}<0.0001$ ) and with IL8 (rho 0.14, $\mathrm{p}<0.001$ ). Furthermore, a positive correlation was also found for PCT with IL6 (rho 0.40, $\mathrm{p}<0.0001$ ), but not with IL8. Correlation between both markers-CRP and PCT—was also significant (rho 0.48, $\mathrm{p}<0.0001)$.

\section{Diagnostic value of markers and cytokines to predict 30-day mortality}

The results of cytokines and markers in the groups of patients who died and of those who survived are displayed in table 3. Significantly higher levels of IL6 IL8, CRP and PCT were found in patients who died within 30 days.

The diagnostic value of markers, cytokines and prognostic scales to predict 30-day mortality was assessed with AUCs,

Table 3 Medians (25th to 75th percentiles) of cytokines and markers in groups with and without mortality

\begin{tabular}{lccl}
\hline & \multicolumn{1}{l}{ Mortality } & \\
\cline { 2 - 3 } Cytokine day $\mathbf{1}$ & Yes & No & p Value \\
\hline CRP $(\mathrm{mg} / \mathrm{dl})$ & $21.9(16.0-32.2)$ & $14.4(7.3-23.5)$ & 0.0001 \\
PCT $(\mathrm{ng} / \mathrm{ml})$ & $1.8(0.63-13.07)$ & $0.58(0.27-2.38)$ & 0.002 \\
$\mathrm{TNF} \alpha(\mathrm{pg} / \mathrm{ml})$ & $44(17-79)$ & $29(17-46)$ & 0.069 \\
IL6 $(\mathrm{pg} / \mathrm{ml})$ & $191(75-982)$ & $73(29-197)$ & 0.0001 \\
IL8 $(\mathrm{pg} / \mathrm{ml})$ & $36(10-65)$ & $6(2-14)$ & 0.0001 \\
IL10 $(\mathrm{pg} / \mathrm{ml})$ & $10(0-65)$ & $7(1-19)$ & 0.25
\end{tabular}

CRP, C-reactive protein; IL, interleukin; PCT, procalcitonin; $\operatorname{TNF} \alpha$, tumour necrosis factor $\alpha$. with the following results: CRP, 0.68 (95\% CI 0.59 to 0.76 ); PCT, 0.66 (0.56 to 0.76); IL6, 0.68 (0.58 to 0.78 ); IL8, 0.76 (0.67 to 0.86 ); IL10, 0.55 (0.44 to 0.68 ); and TNF $\alpha, 0.59$ (0.47 to 0.71 ).

The multivariable study to predict mortality when all cytokines and markers were included found that, after adjustment for PSI and CURB65, the model selected CPR and IL6 as independent predictive variables. The best cut-off point for CRP, using the highest values of sensitivity and specificity after constructing a ROC, was $25 \mathrm{mg} / \mathrm{dl}$. Ninety-nine patients had levels equal to or higher than this level: 38 (38.4\%) developed treatment failure vs $42(13.1 \%)$ of 320 with CRP $<25$; 13 presented shock (14\% vs $17 / 316$ or $5.4 \%$ ); 18 required Intensive Care Unit (ICU) admission (18.2\% vs $27 / 320$ or $8.4 \%$ ); and 7 required mechanical ventilation $(7.1 \%$ vs $12 / 320$ or $3.8 \%)$. The mortality findings in each prognostic scale depending on the threshold of CRP are depicted in table 4.

The AUCs of the different logistic regression models with combinations of markers and cytokines added to any or both prognostic scales were calculated (table 5).

The AUC to predict 30-day mortality significantly increases when the CRP value is added both to the PSI, and to the CURB65 and CRB65 scales. There were increases when IL6 or IL8 were also included (0.86 and 0.87 respectively), but these were not significant when compared with the combination of

Table 4 Mortality according to initial severity, and level of CRP in $\mathrm{mg} / \mathrm{dl}$

\begin{tabular}{lll}
\hline & CRP $<\mathbf{2 5}$ & CRP $\geqslant \mathbf{2 5}$ \\
\hline CRB65 & & \\
$0-1$ & $3 / 200(1.5 \%)$ & $2 / 53(3.77 \%)$ \\
2 & $10 / 90(11.1 \%)$ & $3 / 24(12.5 \%)$ \\
$\geqslant 3$ & $8 / 30(26.6 \%)$ & $9 / 22(40.9 \%)$ \\
CURB65 & & \\
$0-1$ & $1 / 148(0.7 \%)$ & $1 / 41(2.4 \%)$ \\
2 & $4 / 92(4.3 \%)$ & $2 / 26(7.7 \%)$ \\
$\geqslant 3$ & $16 / 80(20 \%)$ & $11 / 32(34.4 \%)$ \\
PSI & & \\
I-II & $0 / 86$ & $0 / 25$ \\
III & $2 / 76(2.6 \%)$ & $0 / 13$ \\
IV & $8 / 114(7 \%)$ & $6 / 39(15.4 \%)$ \\
V & $11 / 44(25 \%)$ & $8 / 22(36.4 \%)$ \\
\hline
\end{tabular}

Deaths/total numbers in each group (\%).

CRB65, Confusion, Respiratory rate, Blood pressure, $\geqslant 65$ years of age; CRP, C-reactive protein; CURB65, Confusion, Urea nitrogen, Respiratory rate, Blood pressure, $\geqslant 65$ years of age; PSI, Pneumonia Severity Index 
CRP alone with the scales. The best AUC (0.88) is achieved with the PSI together with CURB65 and CRP. The diagnostic value of simultaneously using both prognostic scales and CRP with a cut-off point of $25 \mathrm{mg} / \mathrm{ml}$ reaches a sensitivity of 0.77 , a specificity of 0.78 , a positive predictive value of 0.24 and a negative predictive value of 0.97 .

The $\chi^{2}$ goodness-of-fit analysis demonstrated the adequacy of the models $(p>0.05)$.

\section{DISCUSSION}

The most important findings of our study are that the initial value of CRP increases the 30-day mortality prediction by the PSI (AUC from 0.80 to 0.85), by the CURB65 scale (AUC from 0.82 to 0.85 ) and by the CRB65 (AUC from 0.79 to 0.85 ). PCT improves the AUC although without reaching statistical significance. The diagnostic value of CRP and both scales together is the highest, with an AUC of 0.88. Adding the information of cytokines, IL6 and IL8, does not significantly increase the predictive value; thus, in clinical practice they can be substituted, by CRP.

Mortality from CAP is related to the initial severity evaluated with the prognostic scales; however, these have some limitations: PSI better identifies low probability of death, underestimating the risk in young adults, whereas CURB65 better identifies high probability but does not adequately evaluate patient comorbidity. ${ }^{17}$ Niederman ${ }^{17}$ has proposed combining the information of both scales for the assessment of severity and making a decision on whether to admit. In both cases, the scales do not incorporate information of the response of the host against infection, which is a key point to alert clinicians as to the possible development of a more severe evolution and/or complications.

Table 5 Areas under the receiver operating characteristic curve (AUC) of the different models to predict 30-day mortality

\begin{tabular}{|c|c|}
\hline & AUC $(95 \% \mathrm{CI})$ \\
\hline PSI & $0.81(0.75$ to 0.87$)$ \\
\hline PSI+CRP* & $0.85(0.80$ to 0.91$)$ \\
\hline PSI+PCT & 0.83 (0.77 to 0.89$)$ \\
\hline $\mathrm{PSI}+\mathrm{CRP}+\mathrm{PCT}^{*}$ & $0.85(0.79$ to 0.91$)$ \\
\hline $\mathrm{PSI}+\mathrm{CRP}+\mathrm{IL} 6^{*}$ & 0.86 (0.80 to 0.92$)$ \\
\hline PSI+CRP+IL8* & 0.87 (0.82 to 0.93$)$ \\
\hline CRB65 & $0.79(0.72$ to 0.87$)$ \\
\hline CRB65+CRP $\dagger$ & $0.85(0.79$ to 0.90$)$ \\
\hline CRB65+ PCT & $0.83(0.76$ to 0.89$)$ \\
\hline $\mathrm{CRB} 65+\mathrm{CRP}+\mathrm{PCT} \dagger$ & 0.86 (0.80 to 0.92$)$ \\
\hline CRB65+CRP+IL6† & $0.86(0.80$ to 0.92$)$ \\
\hline $\mathrm{CRB} 65+\mathrm{CRP}+\mathrm{IL} 8 \dagger$ & 0.88 (0.81 to 0.93$)$ \\
\hline CURB65 & $0.82(0.76$ to 0.89$)$ \\
\hline CURB65+CRP & 0.86 (0.81 to 0.92$)$ \\
\hline CURB65+PCT & $0.84(0.77$ to 0.90$)$ \\
\hline CURB65+CRP+PCT: & 0.86 (0.79 to 0.92$)$ \\
\hline CURB65+CRP+IL6末 & 0.87 (0.82 to 0.92$)$ \\
\hline CURB-65+CRP+IL8: & 0.88 (0.83 to 0.93$)$ \\
\hline $\mathrm{PSI}+\mathrm{CRB} 65+\mathrm{CRP} * \dagger$ & 0.87 (0.81 to 0.92$)$ \\
\hline $\mathrm{PSI}+\mathrm{CRB} 65+\mathrm{CRP}+\mathrm{IL} 6 * \dagger$ & 0.88 (0.82 to 0.93$)$ \\
\hline PSI+CURB65+CRP* & $0.88(0.83$ to 0.93$)$ \\
\hline PSI+CURB65+CRP+IL6* & 0.88 (0.83 to 0.94$)$ \\
\hline
\end{tabular}

${ }^{*} p<0.05$ for comparison of AUCs with that of PSI.

$\dagger p<0.05$ for comparison of AUCs with that of CRB65.

$\pm \mathrm{p}<0.05$ for comparison of AUCs with that of CURB65.

CRB65, Confusion, Respiratory rate, Blood pressure, $\geqslant 65$ years of age; CRP, C-

reactive protein; CURB65, Confusion, Urea nitrogen, Respiratory rate, Blood pressure,

$\geqslant 65$ years of age; IL, interleukin; PCT, procalcitonin; PSI, Pneumonia Severity Index
An important issue is whether the cytokine profile and markers are merely a reflection of initial severity-that is, if they yield redundant information or if they provide additional information about the host's ability to respond against infection. ${ }^{18}$ In fact, there have been reports of a positive correlation of severity with IL6, IL8 and PCT levels, all three of which we have confirmed. In contrast, CRP did not correlate with PSI, CURB65 or CRB65, as reported by other authors. ${ }^{13}$ Moreover, despite the positive correlation between prognostic scales and cytokines and PCT, it was unsatisfactory, suggesting that the information provided by them is not identical and they might offer complementary data. Thus, higher levels of cytokines $^{19} 20$ and CRP have been demonstrated in patients who subsequently develop shock or severe respiratory failure even with adequate antimicrobial treatment. ${ }^{18}$ In a very interesting study, Kruger et al were able to identify, for the same level of severity in CURB65, patients with higher risk of mortality if PCT was $>0.228 .{ }^{21}$ Christ-Crain et al ${ }^{13}$ also reported that levels of PCT and proadrenomedullin together with PSI improved the AUC for mortality prediction.

In our study we confirm that the initial values of the proinflammatory cytokines IL6 and IL8, together with CRP and PCT were significantly higher in patients with 30-day mortality, similar to previous findings. ${ }^{18} 1922-24$ Furthermore, after stratifying patients according to CRP levels and the three scales, the mortality increased in those with higher levels in those with both a low and a high initial risk of mortality. Multivariable analysis corroborated that, after adjusting for the prognostic scales, CRP and IL6 have an independent predictive value. That is, values of CRP $>25 \mathrm{mg} / \mathrm{dl}$ double the probability of death previously computed in the same class of initial risk. It is worth mentioning that with CRP and both scales together, a very high negative predictive value is reached $(97 \%)$, despite its lower positive predictive value. Chalmers et al ${ }^{25}$ also recently reported that low admission CRP levels were associated with negative predictive value for 30-day mortality, mechanical ventilation, inotropic support and complicated pneumonia. Remarkably, PCT did not significantly improve the prediction of mortality after adjustment for PSI or CURB65, although Christ-Crain $e a^{13}{ }^{13}$ reported a slight increase.

The diagnostic value for 30-day mortality prediction was assessed with the estimation of AUCs obtained with logistic regression models. Although the most explicative mathematic model includes a cytokine (IL6 or IL8) and a marker (CRP), our goal was to find a simpler model with only one marker for easier application in clinical practice. With simpler models with only one marker and one scale, significant improvement was also obtained in the AUCs. Our findings show that CRP significantly improved the diagnostic value of both PSI and the CURB65/CRB65 scales, with a similar gain. Nevertheless, if PCT was included, we found a trend to increase without reaching statistical significance (from 0.80 to 0.82 for PSI, 0.81 to 0.84 for CURB65 and 0.79 to 0.83 for CRB65). A slight increase in the AUC was obtained by simultaneously using both scales and CRP (0.88). The further inclusion of a cytokine in this model did not yield a significant diagnostic improvement. Diagnostic values with cytokines and diagnostic scales are not significantly higher than the values reached with markers, as expected, since release of CRP and PCT is increased by bacterial products, toxins, cytokines and IL6. ${ }^{26}$

One limitation of our study is that outpatients with CAP were not included; therefore, our results cannot be extrapolated to this population. 
In conclusion, the incorporation of the biological markers, PCT and especially CRP, in the PSI and CURB65 prognostic scales might improve their mortality prediction value, to a similar degree for both scales. Measurement of proinflammatory cytokines yields information comparable with that of biological markers. Markers could be used to improve the mortality prediction of the prognosis, based on the inflammatory response of each patient. Further studies are needed to corroborate the additive value of biological markers.

Funding: The study was funded by: CIBER de enfermedades respiratorias (CIBERES), CIBERES is an iniative of ISCIII; FIS 041136; FIS 020616 and Fundació Marató TV3 TV040530. Funding sources provided grants for research not related to any industry and they did not have any role in the design, analysis or interpretation of the results or manuscript approval. An independent statistician, Ivan Arribas, participated in statistical analyses.

Competing interests: AT has been a speaker at a symposium and has received a research grant from Brahms. All other authors have no conflict.

Ethics approval: The study was approved by the Ethics Committees of the two hospitals involved.

\section{REFERENCES}

1. Almirall J, Bolibar I, Vidal J, et al. Epidemiology of community-acquired pneumonia in adults: a population-based study. Eur Respir J 2000;15:757-63.

2. European Respiratory Society. Pneumonia. European Lung White Book 2003:55-65.

3. Armstrong GL, Conn LA, Pinner RW. Trends in infectious disease mortality in the United States during the 20th century. JAMA 1999;281:61-6.

4. Kaplan V, Angus DC, Griffin MF, et al. Hospitalized community-acquired pneumonia in the elderly: age- and sex-related patterns of care and outcome in the United States. Am J Respir Crit Care Med 2002;165:766-72.

5. Mortensen EM, Coley CM, Singer DE, et al. Causes of death for patients with community-acquired pneumonia: results from the Pneumonia Patient Outcomes Research Team cohort study. Arch Intern Med 2002:162:1059-64.

6. Fine MJ, Auble TE, Yealy DM, et al. A prediction rule to identify low-risk patients with community-acquired pneumonia. N Engl J Med 1997;336:243-50.

7. Lim WS, van der Eerden MM, Laing $R$, et al. Defining community acquired pneumonia severity on presentation to hospital: an international derivation and validation study. Thorax 2003;58:377-82.

8. Capelastegui A, Espana PP, Quintana JM, et al. Validation of a predictive rule for the management of community-acquired pneumonia. Eur Respir J 2006;27:151-7.
9. Ewig S, Kleinfeld T, Bauer T, et al. Comparative validation of prognostic rules for community-acquired pneumonia in an elderly population. Eur Respir J 1999;14:370-5.

10. Deng JC, Standiford TJ. The systemic response to lung infection. Clin Chest Med 2005;26:1-9.

11. Nelson S. Novel nonantibiotic therapies for pneumonia: cytokines and host defense Chest 2001;119(2 Suppl):419S-25S.

12. Skerrett SJ, Park DR. Anti-inflammatory treatment of acute and chronic pneumonia Semin Respir Infect 2001;16:76-84.

13. Christ-Crain M, Morgenthaler NG, Stolz D, et al. Pro-adrenomedullin to predict severity and outcome in community-acquired pneumonia [ISRCTN04176397]. Crit Care 2006;10:R96.

14. Lim WS, Lewis S, Macfarlane JT. Severity prediction rules in community acquired pneumonia: a validation study. Thorax 2000;55:219-223.

15. Hanley JA, McNeil BJ. A method of comparing the areas under receiver operating characteristic curves derived from the same cases. Radiology 1983;148:839-43.

16. Hosmer D, Lemeshow S. Applied logistic regression. New York: John Wiley \& Sons, 1989.

17. Niederman MS. Recent advances in community-acquired pneumonia: inpatient and outpatient. Chest 2007:131:1205-15.

18. Menendez R, Cavalcanti M, Reyes S, et al. Markers of treatment failure in hospitalised community acquired pneumonia. Thorax 2008;63:447-52.

19. Fernandez-Serrano S, Dorca J, Coromines M, et al. Molecular inflammatory responses measured in blood of patients with severe community-acquired pneumonia. Clin Diagn Lab Immunol 2003;10:813-20.

20. Schaaf BM, Boehmke F, Esnaashari $H$, et al. Pneumococcal septic shock is associated with the interleukin-10-1082 gene promoter polymorphism. Am J Respir Crit Care Med 2003;168:476-80.

21. Kruger S, Ewig S, Marre R, et al. Procalcitonin predicts patients at low risk of death from community-acquired pneumonia across all CRB-65 classes. Eur Respir J 2008;31:349-55.

22. Monton C, Torres A, El-Ebiary M, et al. Cytokine expression in severe pneumonia: a bronchoalveolar lavage study. Crit Care Med 1999;27:1745-53.

23. Ioanas $\mathbf{M}$, Ferrer $\mathbf{M}$, Cavalcanti $\mathbf{M}$, et al. Causes and predictors of nonresponse to treatment of intensive care unit-acquired pneumonia. Crit Care Med 2004;32: 938-45.

24. Kellum JA, Kong L, Fink MP, et al. Understanding the inflammatory cytokine response in pneumonia and sepsis: results of the Genetic and Inflammatory Markers of Sepsis (GenIMS) Study. Arch Intern Med 2007;167:1655-663.

25. Chalmers JD, Singanayagam A, Hill AT. C-reactive protein is an independent predictor of severity in community-acquired pneumonia. Am J Med 2008;121:219-225.

26. Christ-Crain M, Muller B. Biomarkers in respiratory tract infections: diagnostic guides to antibiotic prescription, prognostic markers and mediators. Eur Respir $J$ 2007; 30:556-73.

\section{Keep up to date: sign up for our alerting services}

Find out automatically when an article is published on a specific topic or by a particular author. We can also alert you when an article is cited or if an eLetter or correction is published. You can also choose to be alerted when a new issue is published online [and when we post articles Online First]. Check out the New Content Alerts and Citation tracker from the Online tools section on the home page. 